\title{
Reflective silicon binary diffraction grating for visible
}

\section{wavelengths}

\author{
Zhen Peng, David A. Fattal, Andrei Faraon, Marco Fiorentino, Jingjing Li, and Raymond G. Beausoleil \\ Hewlett-Packard Laboratories, Palo Alto, CA 94304 USA \\ marco.fiorentino@hp.com
}

\begin{abstract}
We introduce a new device based on sub-wavelength resonant gratings. We built a silicon-on-oxide reflective binary grating for visible light that mimics the functionality of a blazed diffraction grating in a flat geometry.

(C)2011 Optical Society of America
\end{abstract}

OCIS codes: $050.2770,050.6624$

Dielectric gratings made of high index material such as silicon can exhibit internal resonances that profoundly affect their far-field properties [1]. A new type of broadband dielectric mirror has been engineered based on this effect, and has it been shown to perform better than standard dielectric stacks as a top reflector in a Vertical Cavity Surface Emitting Laser (VCSEL) [2]. We recently showed that these gratings have the ability to fully control the phase front of the reflected beam by using a carefully chosen, non-periodic patterning of the grating [3]. Here we use this technology to design reflective diffraction gratings with high efficiency in a particular diffraction order. Our results also supports the idea that very efficient diffractive elements can be realized with lossy grating materials (here, silicon at $650 \mathrm{~nm}$ ) since we show that a $2 \pi$ phase differential can be achieved even for a sub-wavelength grating thickness.

\section{Design and fabrication}

Guided mode resonance (GMR) devices consist of a sub-wavelength binary grating etched in a high-index material designed to be able to guide light. The guided modes are scattered by the grating and, for an appropriate choice of the grating parameters, one can observe resonances in the guided mode. These resonances scatter light back in the free-space modes and therefore provide a way to engineer the grating reflectivity and transmissivity. The phase response associated with the resonantly scattered light can be used to control the phase front of the reflected light [3] and therefore build flat optical devices that act as non-planar mirrors. One would expect that silicon GMR gratings would not work in the visible range because of the strong absorption of silicon at those wavelengths. Figure 1-(a) instead shows that, if one chooses a thin $(180 \mathrm{~nm})$ silicon layer to build the grating, reflectivity in excess of $80 \%$ can be obtained for TM polarized light at $650 \mathrm{~nm}$.
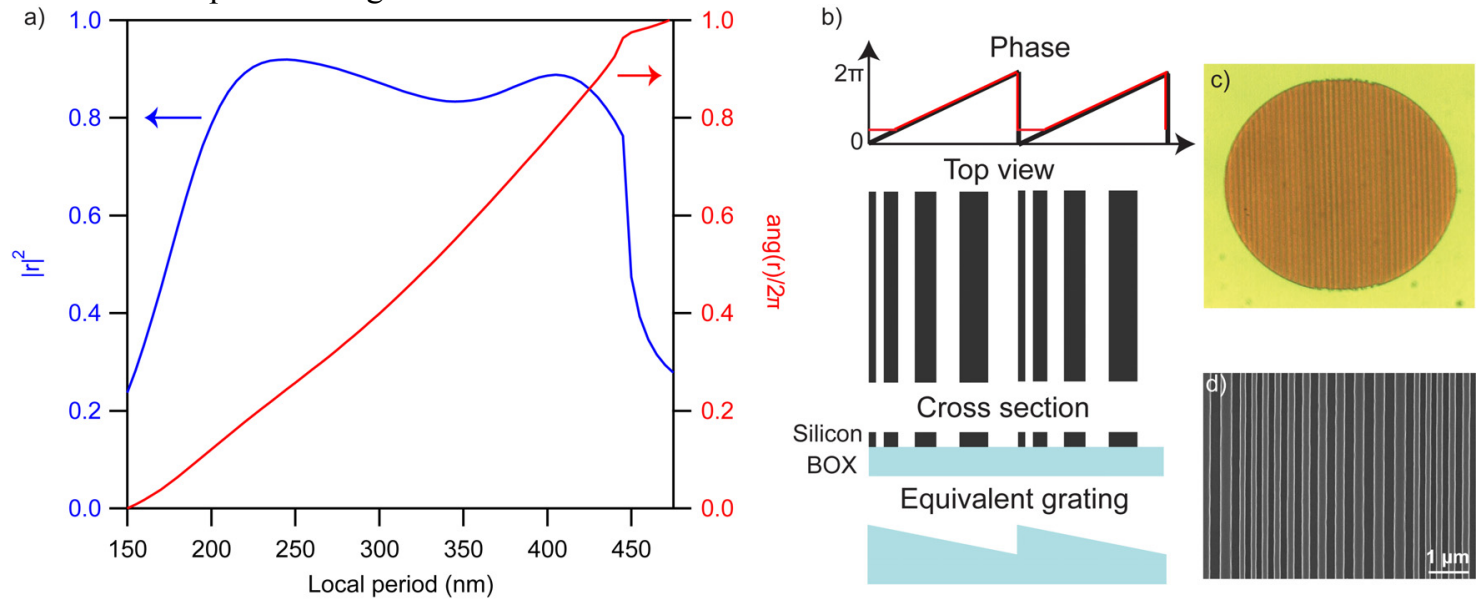

Fig 1: a) Plot of the argument (blue) and normalized phase (red) of the reflection coefficient $r$ of a high contrast mirror as a function of the grating period. Silicon grooves $(\mathrm{n}=3.48,170 \mathrm{~nm}$ thick, duty cycle of $50 \%)$ are placed on quartz substrate $(\mathrm{n}=1.46)$. The design wavelength is $650 \mathrm{~nm}$. $\mathrm{b})$ The top figure shows, in black, the ideal blazed grating phase profile and, in red, the design profile we used. Below the phase profile we have schematic top view and cross section of a binary reflection grating that implements the blazed phase profile. At the bottom the equivalent $3 \mathrm{D}$ grating cross section. c) Microphotograph of a $150 \mu \mathrm{m}$ grating d) SEM picture of the grating grooves showing the modulation in the period. 
Standard blazed diffraction gratings use a 3D groove shape to achieve the right phase profile (see bottom picture of Fig.1-(b)). Blazed gratings are more complex to fabricate than binary gratings, and suffer from shadowing effects which limits their practical efficiencies. Using the spatial modulation of GMRs we can build a phase profile that mimics an optimal blazed grating for the first diffracted order. We designed a grating operating with TM polarization at $650 \mathrm{~nm}$ wavelength with a first order diffraction angle $\theta_{1}=10^{\circ}$. Using crystalline silicon, the optimum grating thickness was found to be in the range $160-180 \mathrm{~nm}$, thin enough to greatly reduce the effects of silicon absorption. Due to a limitation in the fabrication capabilities we decided to design a grating that has the phase profile shown by the red curve in the top graph of Fig.1-(b). The "flat" portion of the grating contributes to diffraction in the zeroth order of the grating (specular reflection). This effect can be observed in the table in Fig 2.

\section{Experimental results}

Figure 2 shows the results of the grating scattering at $10^{\circ}$ angle of incidence measured using a spectroscopic ellipsometer. The main plot shows the light intensity versus the scattering angle (measured relative to specular reflection). The light is strongly scattered in the first order with a weaker scattering peak at the second order around $20^{\circ}$. Observe that, close to the design wavelength of $650 \mathrm{~nm}$ the second order scattering is effectively suppressed while the first order has maximum intensity. The inset of Fig. 2 shows the change in the reflection angle as a function of the incident wavelength. The grating dispersion is equivalent to that of a grating of period $3.4 \mu \mathrm{m}$, this is in agreement with the period of the super-structure shown in Fig. 1(d).

To measure the power scatters in all diffraction orders so we used a laser $(\lambda=640 \mathrm{~nm})$ at an angle of incidence of $\sim 5^{\circ}$. The table in Fig 2 shows the result of the measurement. As expected the largest amount of power is directed in the first order with some power left in the zeroth order due to the configuration of the grating. The other orders combined contain approximately $5 \%$ of the power.

We have introduced an application of GMR to blazed binary diffraction gratings. These gratings are fabricated a straightforward lithographic process. Given the flexibility of the fabrication technique it is also possible to build curved diffraction gratings with high numerical apertures with a process that is identical to the one used for a flat gratings. In addition we have shown that silicon GMRs can be used for visible wavelengths, provided that the gratings parameters are appropriately chosen.

\section{References}

[1] S. S. Wang, R. Magnusson, J. S. Bagby, and M. G. Moharam, "Guided-mode resonances in planar dielectric-layer diffraction gratings," J. Opt. Soc. Am. A 7, 1470-1474 (1990).

[2] M. Huang, Y. Zhou, and C. Chang-Hasnain, “A surface-emitting laser incorporating a high-index-contrast subwavelength grating," Nature Photon. 1, 119-122 (2007).

[3] D. Fattal, J. Li, Z. Peng, M. Fiorentino, and R. G. Beausoleil, "Flat dielectric grating reflectors with focusing abilities," Nature Photon. 4, 466-470 (2010).

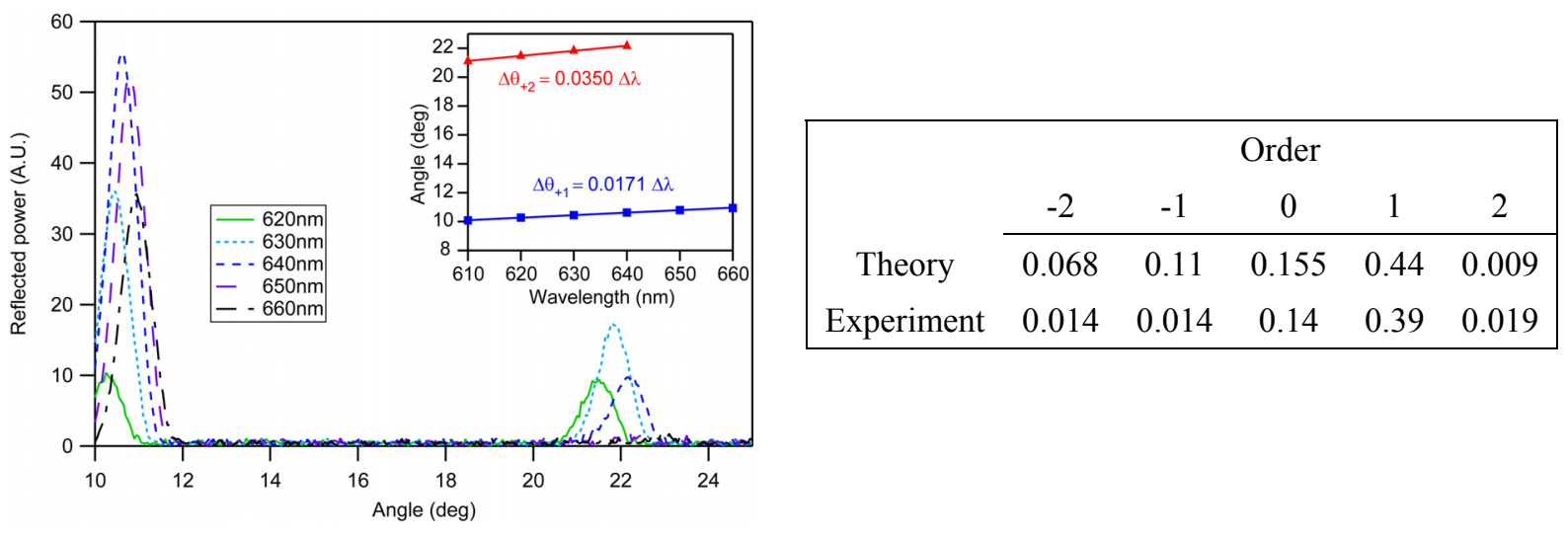

Fig 2: Plot of the reflectivity versus scatter angle for the binary reflection grating at an AOI of $10^{\circ}$ and various wavelengths. The angles on the abscissas are measured relative to the specular reflection angle. In the inset, plot of the reflection angle as a function of the test wavelength for the first (blue squares) and second (red triangles) diffraction order. The lines are linear fits to the data. The table on the right shows the theoretical and experimental power distribution among diffraction orders. 